\title{
Auditory Brainstem Responses in the C57BL/6J Fragile X Syndrome Knockout Mouse Model
}

\author{
Amita Chawla ${ }^{1}$, Elizabeth A McCullagh ${ }^{*}$
}

${ }^{1}$ Department of Integrative Biology, Oklahoma State University, Stillwater, OK, USA

*Correspondence:

Corresponding Author

elizabeth.mccullagh@okstate.edu

Keywords: Auditory Brainstem Response (ABR), Fragile X Syndrome, binaural hearing, sex differences, mouse model

\section{Abstract (250 words)}

Sensory hypersensitivity, especially in the auditory system, is a common symptom in Fragile $\mathrm{X}$ Syndrome (FXS), the most common monogenic form of intellectual disability. However, linking phenotypes across genetic background strains of mouse models has been a challenge and could underly some of the issues with translatability of drug studies to the human condition. This study is the first to characterize the auditory brainstem response (ABR), a minimally invasive physiological readout of early auditory processing that is also used in humans, in a commonly used mouse background strain model of FXS, C57BL/6J. We measured morphological features of pinna and head and used ABR to measure hearing range, monaural and binaural auditory responses in hemizygous males, homozygous females and heterozygous females compared to wildtype mice. Consistent with previous work we showed no difference in morphological parameters across genotypes or sexes. Male FXS mice had increased threshold for high frequency hearing at $64 \mathrm{kHz}$ compared to wildtype males, while females had no difference in hearing range between genotypes. In contrast, female homozygous FXS mice had decreased amplitude of wave IV of the monaural ABR, while there was no difference in males for amplitudes and no change in latency of ABR waveforms across sexes and genotypes. Lastly, FXS males had increased latency of the binaural interaction component (BIC) at 0 ITD compared to wildtype males. These findings further clarify auditory brainstem processing in FXS by adding more information across genetic background strains allowing for a better understanding of shared phenotypes.

Fragile X Syndrome (FXS) is the most common monogenic form of autism spectrum disorder (ASD) and shares many attributes of ASDs including auditory hypersensitivity and other sensory disruptions (Abbeduto and Hagerman, 1997; Chen and Toth, 2001; Hagerman and Hagerman, 2002; Arnett et al., 2014). FXS is a tractable genetic model for ASD with several commercially available models, including rat and mouse (The Dutch-Belgian Fragile X Consorthium et al., 1994; Till et al., 2015; Tian et al., 2017). Despite the common use of these models to study FXS, phenotypes are not always shared between species and background strains, particularly for sensory processing. As a result, drug therapies have struggled to rescue the human disorder (Dahlhaus, 2018). One of the most common symptoms described in people with FXS and ASD is auditory hypersensitivity (Ethridge et al., 2017; Stefanelli et al., 2020). The mechanisms that underly auditory alterations are unknown, but likely 

cortex across sexes, background strains, and models is needed to fully understand shared phenotypes and circuitry involved in this common symptom.

The auditory brainstem is one brain region in the ascending auditory pathway that has shown to have anatomical, physiological, and behavioral alterations in FXS mouse models (Brown et al., 2010; Beebe et al., 2014; Wang et al., 2014, 2015; Rotschafer et al., 2015; Garcia-Pino et al., 2017; McCullagh et al., 2017, 2020a; Rotschafer and Cramer, 2017; Curry et al., 2018; El-Hassar et al., 2019; $\mathrm{Lu}, 2019$ ). The auditory brainstem is the first site of binaural processing of sound location in the brain using interaural timing and level differences (ITD and ILD respectively) to compute sound source locations (Grothe et al., 2010). This brain area is also involved in separating spatial channels allowing for complex listening environments. Disruptions in this spatial separation and binaural processing could lead to auditory hypersensitivity due to inability to separate sound sources (Bronkhorst, 2015). One measure of auditory brainstem physiology, and binaural hearing, that can be directly translated between animal models and humans is the auditory brainstem response (ABR)

53 (Laumen et al., 2016).

The ABR is a minimally invasive physiological measure that allows for simultaneous assessment of sound processing across multiple brainstem nuclei, as each wave of the ABR directly corresponds to distinct areas of the ascending auditory brainstem pathway. These features make the ABR an attractive translational tool. Indeed, recent evidence suggests that ABR measurements are an early indicator for auditory dysfunction in ASD (Santos et al., 2017). ABRs can also be used to assess binaural hearing, which is essential for sound localization and hearing in noisy environments and often impaired in ASD (Visser et al., 2013). Monoaural ABRs can be recorded by stimulating each ear separately and binaural responses can be generated by stimulating both ears simultaneously. The sum of the two monaural (left and right) responses should equal the binaural (both ear) response since the recruited neural activity from each ear should be double when stimulated simultaneously. However, this is not the case, there is a difference that arises when the summed monoaural responses are subtracted from the binaural response, called the binaural interaction component (BIC). The BIC is thought to be a direct measure of binaural processing ability in humans and animals that requires the precise balance of excitatory and inhibitory drive in brainstem sound localization circuits (Laumen et al., 2016).

In this study we report on the hearing ability, using the ABR and morphological craniofacial and pinna features, of the most common mouse model of FXS, C57BL/6J across the sexes and females heterozygous for the Fmr1 mutation. We hypothesize that there may be sex differences in ABRs independent of FXS genotype, but that additionally FXS animals are likely to have alterations in peak amplitude or latency of ABRs and impaired high frequency hearing compared to wildtype consistent with work in other FXS mouse strains (Kim et al., 2013; Rotschafer et al., 2015; El-Hassar et al., 2019). Establishing core auditory phenotypes across the sexes and different mouse strains is key to creating a toolbox of techniques that may translate to human FXS.

\section{Materials and Methods}

All experiments complied with all applicable laws, National Institutes of Health guidelines, and were

79 approved by the Oklahoma State University IACUC. 
Experiments were conducted in C57BL/6J (stock \#000664, B6) wildtype background, hemizygous male, homozygous, or heterozygous female $F m r 1$ mutant mice (B6.129P2- $F m r 1^{t m} 1 \mathrm{Cgr} / \mathrm{J}$ stock \#003025, Fmr1 or Fmr1 het respectively) obtained from the Jackson Laboratory (Bar Harbor, ME USA)(The Dutch-Belgian Fragile X Consorthium et al., 1994). Sex was treated as a biological variable and differences between the sexes, when present, are noted in the results. Numbers of animals for each experiment used are listed in the figure legends and ranged from 6-10 animals per sex and genotype. Animals ranged in age from $62-120$ days old (average ages per genotype $89 \pm 4$ days old B6, $101 \pm 3$ days old Fmr1, and $97 \pm 4$ days old Fmr1 het).

\subsection{Morphological Measures}

Features of animal's head, pinna, and body mass (weight) were measured for each genotype using 6 Inch Stainless Steel Electronic Vernier Calipers (DIGI-Science Accumatic digital caliper Gyros Precision Tools Monsey, NY, USA) and an electronic scale. The distance between the two pinnae (inter pinna distance), distance from the nose to the middle of the pinna (nose to pinna distance) and pinna width and length were measured (Figure 2A). The effective diameter was calculated as the square root of pinna length times pinna width (Anbuhl et al., 2017).

\subsection{ABRs}

ABR recordings were performed using similar methods to previously published work (Benichoux et al., 2018; McCullagh et al., 2020a; New et al., 2021). Animals were anesthetized using two mixtures of ketamine-xylazine $60 \mathrm{mg} / \mathrm{kg}$ ketamine and $10 \mathrm{mg} / \mathrm{kg}$ xylazine for initial induction followed by maintenance doses of $25 \mathrm{mg} / \mathrm{kg}$ ketamine and $12 \mathrm{mg} / \mathrm{kg}$ xylazine. Once anesthesia was confirmed by lack of a toe-pinch reflex, animals were transferred to a small sound attenuating chamber (Noise Barriers Lake Forest, IL, USA) and body temperature was maintained using a water-pump heating pad. Subdermal needle electrodes were placed under the skin between the ears (apex), directly behind the apex in the nape (reference), and in the back leg for ground. This montage has been shown to be particularly effective in generating the BIC (Levine, 1981; Laumen et al., 2016). Evoked potentials from subdermal needle electrodes were acquired and amplified using a Tucker-Davis Technologies (TDT, Alachua, FL, USA) RA4LI head stage and a TDT RA16PA preamplifier. Further amplification was provided by a TDT Multi I/O processor RZ5 connected to a PC with custom Python software for data recording. Data were averaged across 500-1000 repetitions per condition and processed using a second order $50-3000 \mathrm{~Hz}$ filter over $12 \mathrm{~ms}$ of recording time.

Sound stimuli (see below for varying types) were presented to the animal through TDT EC-1 electrostatic speakers (frequencies $32-64 \mathrm{kHz}$ ) or TDT MF-1 multi-field speakers (frequencies 1 $24 \mathrm{kHz}$ and clicks) coupled through custom ear pieces fitted with Etymotic ER-7C probe microphones (Etymotic Research Inc, Elk Grove Village, IL, USA) for in-ear calibration (Beutelmann et al., 2015). Sounds were generated using a TDT RP2.1 Real-Time processor controlled by custom Python code at a sampling rate of $48828.125 \mathrm{~Hz}$. Sounds were presented at an interstimulus interval of $30 \mathrm{~ms}$ with a standard deviation of $5 \mathrm{~ms}$ (Laumen et al., 2016). An additional

118 rejection threshold was set to eliminate high amplitude heart rate responses from average traces and 119 improve signal to noise ratio.

\subsubsection{Audiogram}

121 Hearing range of animals was tested using the threshold for hearing across different frequencies of 122 sound $(1,2,4,8,16,24,32,46,64 \mathrm{kHz})$. Threshold was determined using a visual detection method 
123 (Brittan-Powell and Dooling, 2004), or the lowest level (dB SPL) a response could be detected 124 (independent of wave) or $2.5 \mathrm{~dB}$ SPL below the lowest level that elicited a response. Audiogram 125 stimuli consisted of tone bursts ( $2 \mathrm{~ms} \pm 1 \mathrm{~ms}$ on/off ramps) of varying frequency and intensity.

\section{$126 \quad$ 2.3.2 Monaural ABRs}

127 Click stimuli (0.1 ms transient) were presented to each ear independently to generate monaural evoked potentials. Peak amplitude (voltage from peak to trough) and latency (time to peak amplitude) were measured across the four peaks of the ABR waveform at $90 \mathrm{~dB}$ SPL (Figure 1A). The trough was considered the lowest point for that wave. Monaural data from the two ears were averaged to determine monaural amplitude and latency for each animal. Like hearing thresholds across

132 frequency, click threshold was determined for each genotype and sex. Click threshold is determined

133 by decreasing intensity of sound in 5 - $10 \mathrm{~dB}$ SPL steps until ABR waveforms disappear.

\section{$134 \quad$ 2.3.3 Binaural ABRs}

135 Click stimuli at $90 \mathrm{~dB}$ SPL were also presented to both ears simultaneously to generate a binaural 136 evoked potential. The binaural interaction component (BIC) of the ABR was calculated by 137 subtracting the sum of the two monaural ABRs from the binaural ABR (Laumen et al., 2016; 138 Benichoux et al., 2018)(Figure 1B and C). BIC amplitude and latency were then measured using

A

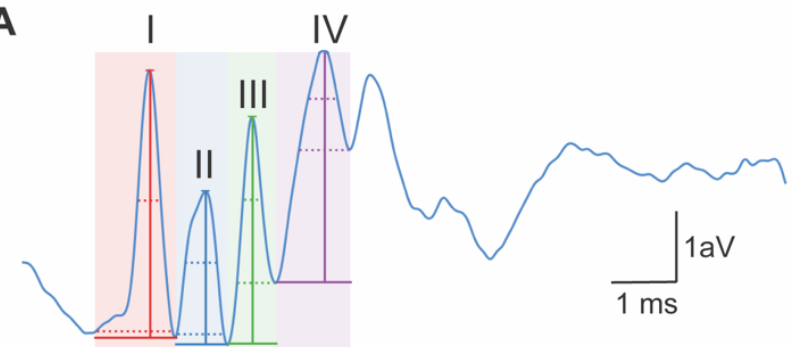

B
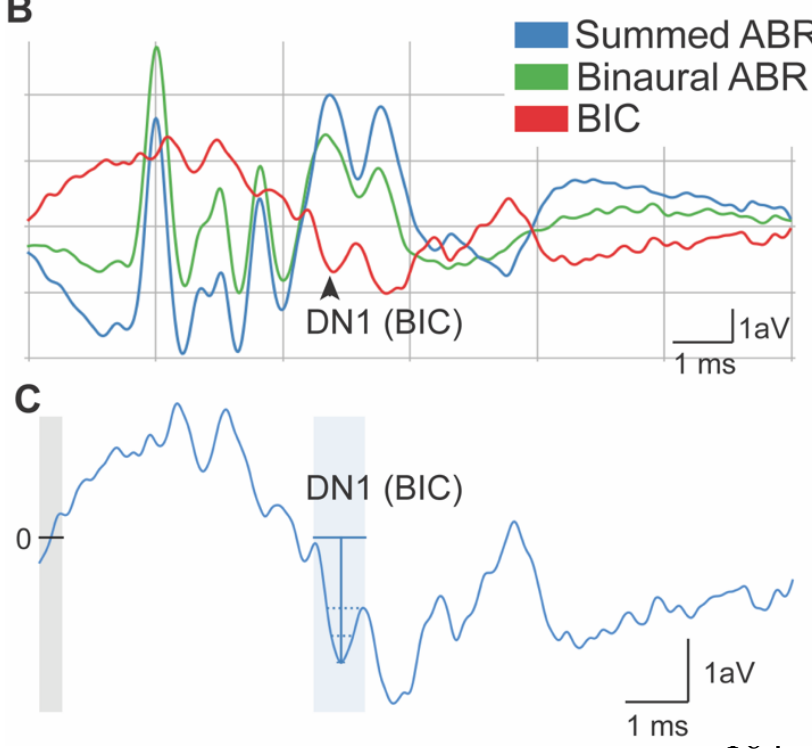
custom Python software, with amplitude being relative to the zero baseline of the measurement (Figure 1C, gray area with line). BIC was characterized as the prominent negative DN1 wave corresponding to the fourth wave of the binaural and summed ABR (Figure 1B). To measure interaural timing difference (ITD) computation using the BIC, animals were presented with stimuli that had varying ITDs of $\pm 2 \mathrm{~ms}$ in $0.5 \mathrm{~ms}$ steps and corresponding BIC amplitudes and latencies were calculated like above. This ITD range was chosen to be comparable to other studies in small rodents (Benichoux et al., 2018).

Figure 1. Quantification of ABR signals. Monaural ABR amplitudes were quantified for each ear as the voltage between the peak of the ABR and trough of the waveform for waves I-IV (A). Latency was calculated as the time when the height of the peak occurred. DN1 or $\mathrm{BIC}$ (red) was calculated as the prominent negative peak corresponding with wave IV of the summed (blue) and binaural (green)(B). BIC is calculated as the summed $A B R$ subtracted from the binaural $A B R$. The $\mathrm{BIC}$ amplitude was calculated as voltage at the peak of the DN1 waveform to the baseline (0, line and gray area) of the measurement $(\mathrm{C})$. Scale represents 1 voltage unit $(\mathrm{Y})$ during $1 \mathrm{~ms}(\mathrm{X})$.

\subsection{Analysis of ABR waveforms}

166 Custom python software was used to analyze evoked potentials for monaural and binaural stimuli 167 (New et al., 2021). To account for fluctuation in the baseline signal of the ABR, raw traces were 
zeroed to establish a baseline across traces. The software included automatic peak detection with the capability of manual correction or deselection upon visual confirmation.

\subsection{Statistical analyses}

171 Figures were generated using R Studio (R Core Team, 2013), ggplot2 (Wickham, 2016), and Adobe 172 Illustrator (Adobe, San Jose, CA USA). Data points on Figures 3, 4, and 5 represent means and error 173 bars reflect standard error, boxplots in Figure 2 display the median and $25^{\text {th }}-75^{\text {th }}$ percentiles (or $1^{\text {st }}$ and $3^{\text {rd }}$ quartiles respectively) the whiskers represent $+/-1.5$ times the interquartile range. Data that falls outside the range are plotted as individual points. Multivariate data (monaural peak amplitude and latency, audiogram, and BIC amplitude and latency across ITD) were analyzed using linear mixed effects (lme4) models (Bates et al., 2015) with sex, genotype, and condition (ITD, Frequency,

A

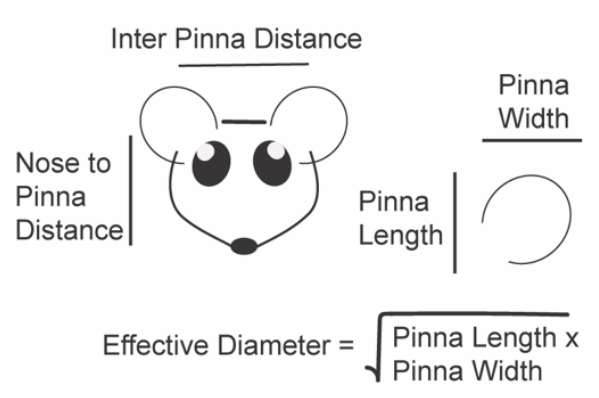

C

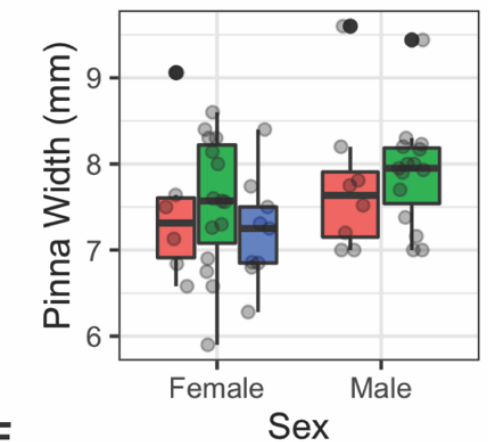

E

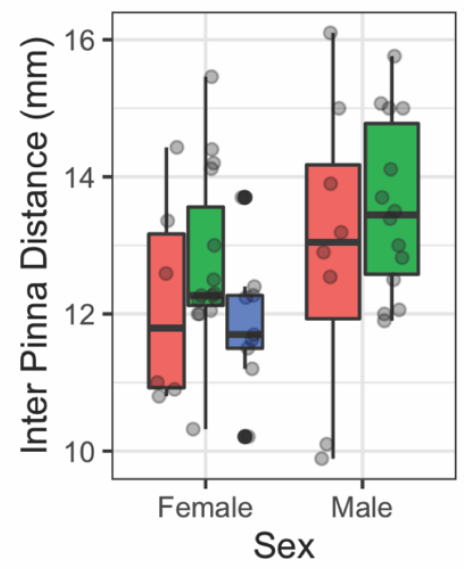

B

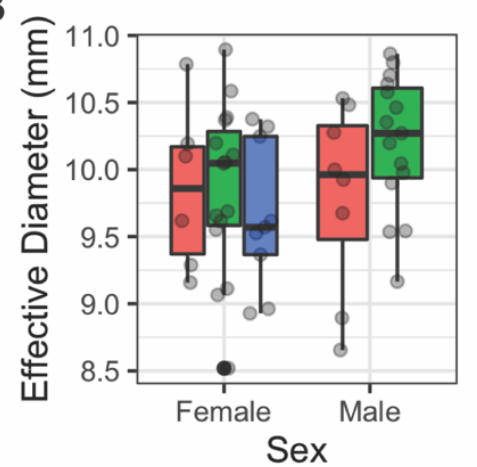

D

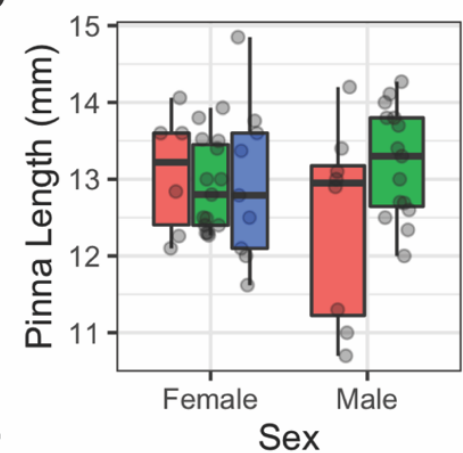

$\mathbf{F}$

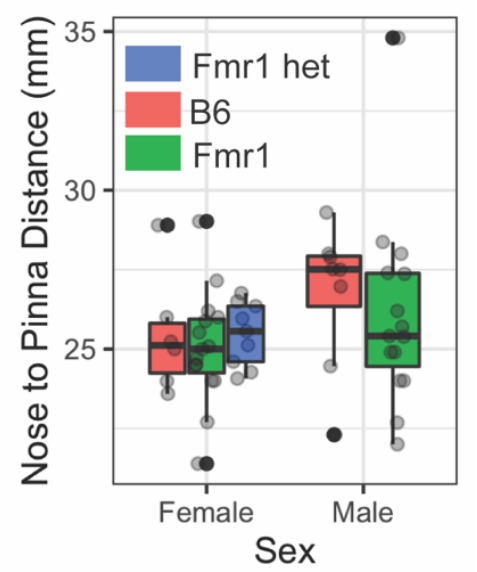

Peak) as fixed effects and animal as a random effect. It was expected that there may be differences between the sexes and genotypes therefore a priori, it was determined that estimated marginal means (emmeans; (Lenth, 2019)) would be used for pairwise comparisons between sexes and genotype. Two-way ANOVAs were performed to compare relationships between morphological features, sex, and genotype with adjusted Tukey posthoc analysis to compare groups. Where values are indicated as statistically significant between the two genotypes, $*$ indicated a $\mathrm{p}$ value of $<0.05$, ** $=\mathrm{p}<0.01$, and $* * *=\mathrm{p}<0.0001$.

Figure 2. Morphological features of FXS mice. Pinna and head features (A) were measured between the sexes (x-axis) and genotypes (purple = B6, teal $=$ Fmr1, yellow = Fmr1 het). There was no difference between the sexes or genotypes for any of the measures (effective diameter $B$, pinna width $C$, pinna length $D$, inter pinna length $E$, or nose to pinna length $F$ ). Data represent $6 \mathrm{~B} 6,15 \mathrm{Fmr} 1,9 \mathrm{Fmr} 1$ het females and $8 \mathrm{~B} 6,15 \mathrm{Fmr} 1$ males.

\section{$4 \quad$ Results}

211 We used both morphological and physiological features to examine hearing differences in a

212 commonly used mouse model of FXS, C57BL/6J across genotypes and sexes. Hearing measurements 
213 included frequency hearing range, monaural hearing ability, and binaural processing using the ABR,

214 while morphological features included pinna and head measurements.

\section{$215 \quad 4.1$ Morphological features}

216 People with FXS have altered craniofacial features, including large ears (Loesch et al., 1988).

217 Consistent with our previous work (McCullagh et al., 2020a) we see no difference between B6,

218 Fmr1, or Fmr1 het animals for pinna attributes (Figure 2C pinna width, 2D pinna length, 2B effective diameter). In addition, pinna characteristics were the same between the sexes $(p=0.175$ pinna width, $\mathrm{p}=0.96$ pinna length, $\mathrm{p}=0.267$ effective diameter Figure $2 \mathrm{~B}-\mathrm{D})$. When genotypes were compared within the same sex, there were no differences in weight, but sexes were significantly different independent of genotype $(p=0.0023)$ with females weighing significantly less than males. Similar to pinna morphology, there was no significant difference in either distance between pinna or distance from the nose to pinna between the genotypes or sexes (Figure $2 \mathrm{E}$ and $\mathrm{F}$ ). These data suggest that
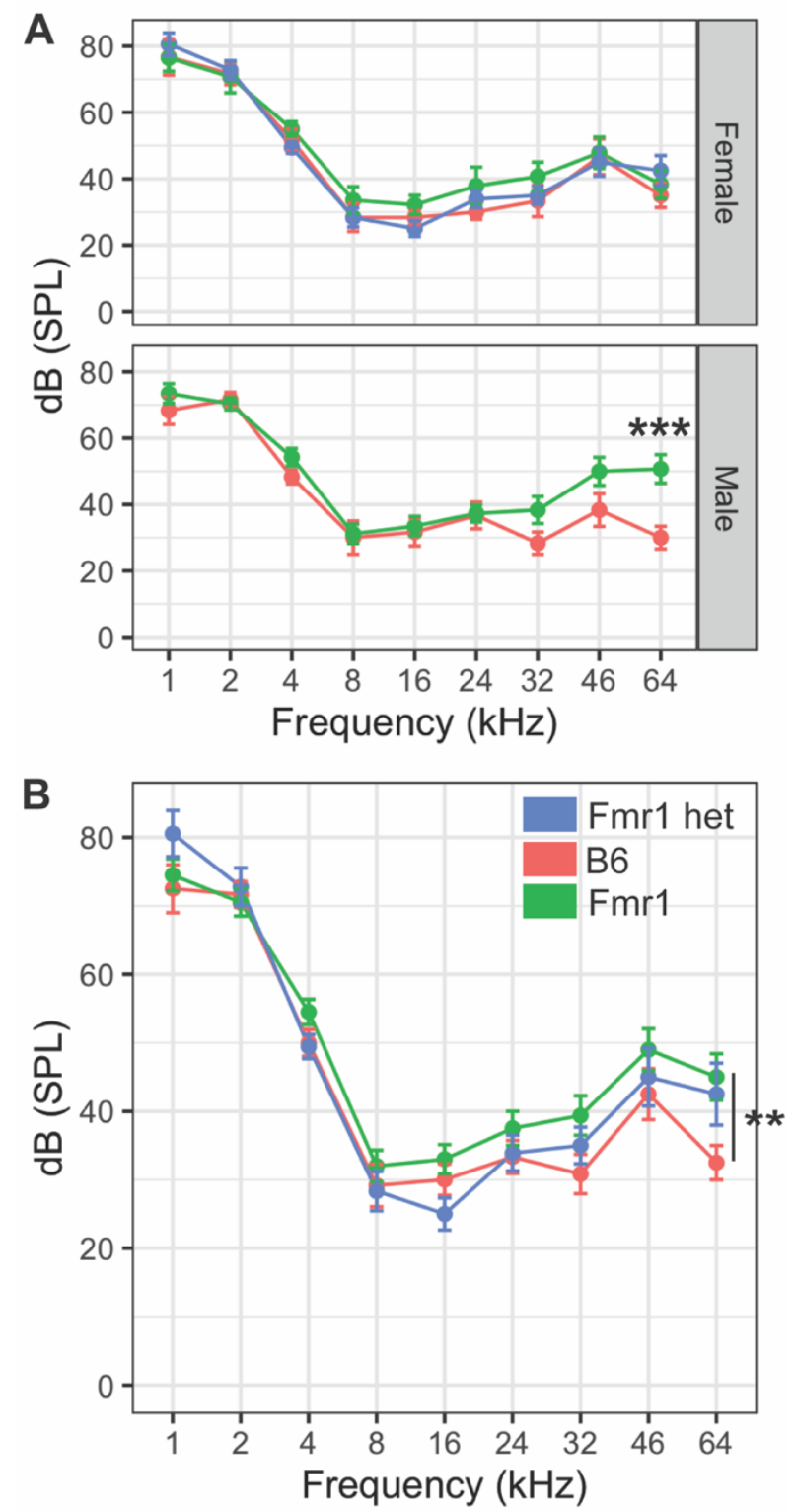
mice do not share the same craniofacial changes, at least in the measurements described here, as people with FXS.

Figure 3. Hearing range of FXS mice. Hearing threshold (dB SPL) was measured across frequencies (1$64 \mathrm{kHz}$ ) in male and female mice of all genotypes (A). Fmr1 (green) males (lower panel) had significantly higher threshold hearing at $64 \mathrm{kHz}$ compared to $\mathrm{B} 6$ (red) males. There were no differences in hearing range between female Fmr1 (green), B6 (red), and Fmr1 het (blue) mice (top panel A). When sexes were combined, the significant difference in hearing at $64 \mathrm{kHz}$ persisted in Fmr1 animals compared to B6 but was not significant in female Fmr1 het animals (B). ${ }^{* *}=p<0.01,{ }^{* * *}=p<$ 0.001 . Data represent $6 \mathrm{~B} 6,7 \mathrm{Fmr} 1,9 \mathrm{Fmr} 1$ het females and 6 B6, 11 Fmr1 males.

\subsection{Hearing range}

Our previous work showed that Fmr1 mice have increased thresholds for high frequency hearing compared to B6 at $16 \mathrm{kHz}$ (McCullagh et al., 2020a). However, that work was limited by measuring only three frequencies $(4,8,16 \mathrm{kHz})$ and seven mice of each genotype (combined sexes). Mice hear much higher frequencies than humans (Radziwon et al., 2009), therefore we wanted to measure whether this high frequency hearing loss exists across the frequencies in which mice hear in Fmrl mutants and with a more in-depth sex specific analysis. Interestingly, there were no differences between genotypes across most of the frequencies tested except for an increase in hearing threshold at $64 \mathrm{kHz}$ in Fmr1 mice compared to B6 (Figure 3B). There were no significant differences in hearing range between the sexes, but Fmr1 males did have significantly higher threshold at $64 \mathrm{kHz}$ than 
female Fmr1 mice suggesting that the phenotype is mostly driven by males $(p=0.0317)$ and indeed both female Fmr1 and female Fmr1 het mice were not different than B6 females (Figure 3A). Best frequencies for both genotypes, as indicated by lower threshold, of mice were between $8-64 \mathrm{kHz}$ consistent with specialized high frequency hearing.
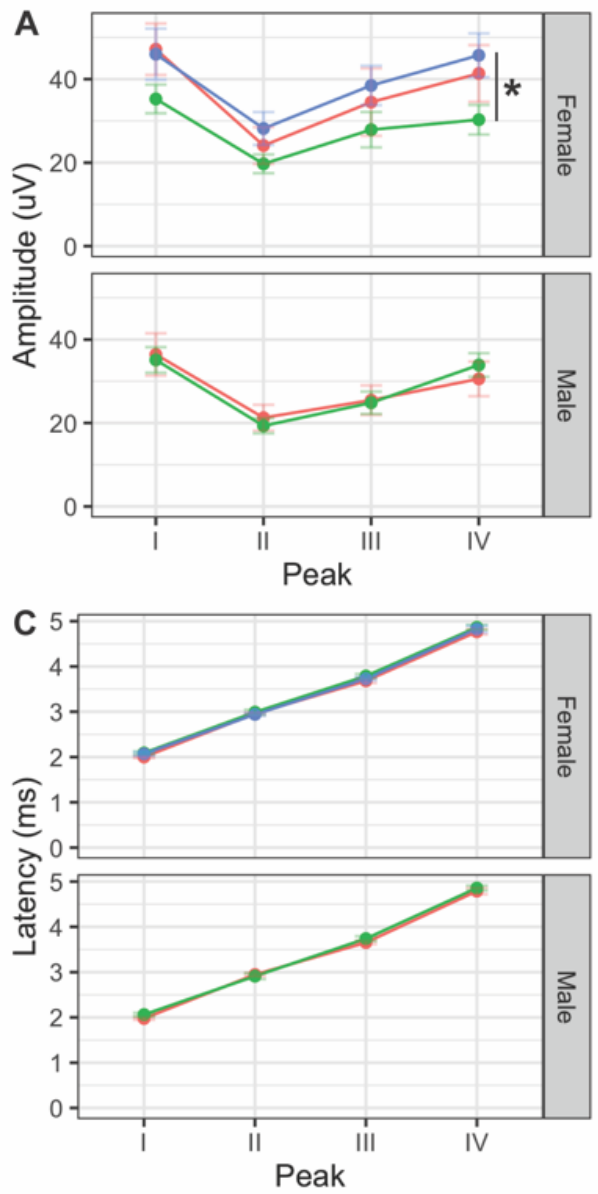
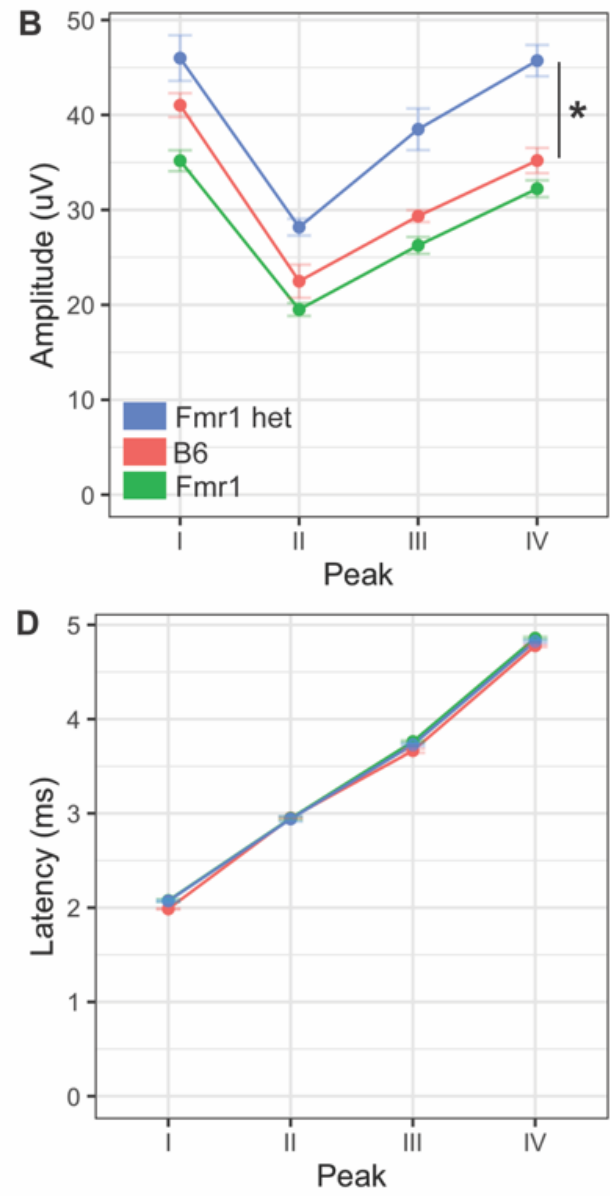

Figure 4. Monaural hearing in FXS mice. Monaural amplitudes and latencies for peaks I-IV of the ABR were recorded for Fmr1, Fmr1 het, and B6 animals. Peak IV amplitude was significantly lower in Fmr1 mice females compared to Fmr1 het females (A upper), There were no significant differences in amplitudes for males (A, lower). When combined, there was a significant difference in Fmr1 het animals compared to Fmr1 (B). There was no difference in latency of peaks I-IV between sexes $(C)$ or genotypes (D). ${ }^{*}=p<$ 0.05 . Data represent 6 B6, 12 Fmr1, 9 Fmr1 het females and 8 B6, 14 Fmr1 males.

\subsection{Monaural hearing}

Amplitude and latency of monaural ABRs correspond with neural activity across the ascending auditory pathway, with each wave representing different brain areas involved in auditory processing (Alvarado et al., 2012). Other studies have shown both latency and amplitude alterations in the FVB mouse strain of Fmr1 mutation (Kim et al., 2013; Rotschafer et al., 2015; El-Hassar et al., 2019). We measured ABR responses of Fmr1 mutants to monaural click stimuli compared to B6 mutant mice to determine if they have a similar ABR phenotype to the FVB strain. We saw no differences in overall click threshold for either genotype or sex $(p=0.102$ genotype and $p=0.47$ for sex $)$. Amplitude of monaural responses was significantly lower for wave IV of the ABR in Fmr1 females compared to Fmr1 het females (Figure 4A upper). Indeed, Fmr1 het female amplitudes were closer to B6 than Fmr1 females, though Fmr1 females were not significantly different from B6. In contrast, Fmr1 male amplitudes for waves I-IV were not different from B6 (Figure 4A lower). When sexes were combined, Fmr1 het females had significantly higher amplitudes than B6, and were close to being significantly higher than Fmr1 mice $(p=0.0593)$. Consistent with sex driving the differences in genotype, peak amplitudes varied between the sexes. Female B6 mice had significantly higher amplitude peaks I and IV compared to B6 males $(p=0.0295$ peak I and $p=0.0289$ peak IV). In contrast, there were no sex differences between male and female Fmr1 mice suggesting a more male-like phenotype (independent of genotype) in homozygous Fmrl females. There were no differences between the sexes or genotypes in latency of monaural peaks (Figure 4C and D). 


\subsection{Binaural hearing}

311 While the monaural ABR provides information about binaural areas of the brainstem (potentially 312 peaks III and IV), since they are elicited by either sound played directly to one ear (closed field) or 313 equally to both ears (open field), little information can be gained about binaural integration of sound 314 information. We used the BIC of the ABR to measure binaural processing ability of the brainstem as the BIC varies with ITDs played to both ears. We saw no differences in amplitude of the BIC at any ITD between the two genotypes $(p=0.809)$ or with sex $(p=0.6904$, Figure $5 A, B)$, though there was a significant difference between Fmrl male and female mouse BIC amplitudes at $1.5 \mathrm{~ms}$ ITD.

318 Latency of the BIC was significantly slower in male Fmr1 compared to B6 (Figure 5C, lower panel) only at 0 ITD, with no difference in genotype for female mice (Figure 5C, upper panel). When data were combined for sexes across genotype, there was no significant difference in latency of the BIC at any ITD (Figure 5D). There were differences in latency of the BIC between B6 (-1.5 ms) and Fmr1 ( $1 \mathrm{~ms})$ males and females though there was no overall main effect of $\operatorname{sex}(p=0.3367)$.
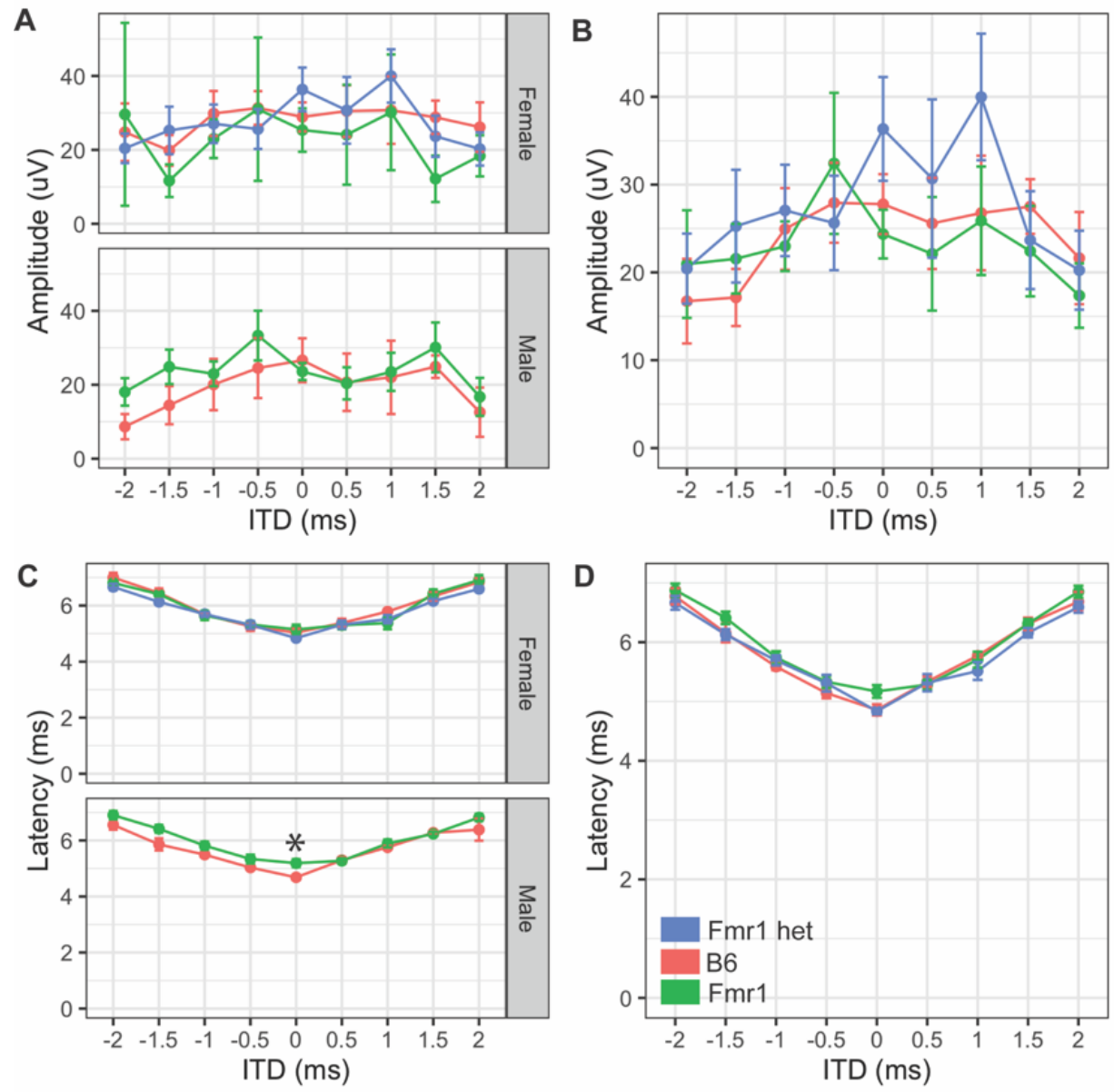

Figure 5. Binaural hearing in FXS mice. Binaural amplitudes and latencies for the BIC at ITDs between -2 to $+2 \mathrm{~ms}$ in $0.5 \mathrm{~ms}$ steps were recorded for Fmr1 (green), Fmr1 het (blue), and B6 (red) animals. No differences in amplitude of the BIC with ITD for females (A upper), or males (A lower). When sexes were combined, there was no significant difference in amplitude of the BIC with ITD (B). Fmr1 males had significantly longer latency of the BIC at 0 ITD compared to $B 6$ males (C, lower), while there was no difference in latency of female responses (C, upper). When sexes were combined, there was no difference in the BIC latency across ITDs between the genotypes (D). ${ }^{*}=p<$ 0.05 . Data represent $6 \mathrm{~B} 6,7$ Fmr1, 9 Fmr1 het females and $6 \mathrm{~B} 6,9 \mathrm{Fmr} 1$ males.

\section{Discussion}

354 This is the first study to characterize the ABR in the C57BL/6J Fmr1 mutant mouse, and in particular highlights morphological characteristics, hearing range, monaural ABRs, and binaural integration across sexes and in heterozygote females. Consistent with previous work, we see an increase in 
hearing threshold at high frequencies in Fmr1 mice, though this phenotype is male specific, and no change in morphology (pinna or facial characteristics)(McCullagh et al., 2020a). Female Fmr1 mice have reduced wave IV amplitudes of the monaural ABR, and wildtype females have increased wave I and IV amplitudes compared to B6 males, suggesting that female Fmr1 mice have a more male-like phenotype for monaural ABR amplitude. Lastly, we showed that male Fmr1 mice have increased latency of the BIC at 0 ITD, but not other ITDs or changes in amplitude of the BIC across ITD compared to B6 animals suggesting changes in timing of the processing of binaural information that does not change overall ITD following ability.

Pinnae size and shape are the first feature available to determine sound localization ability in animals with external ears (Butler, 1975; Musicant and Butler, 1984). Craniofacial alterations including prominent ears and elongated face are hallmark features of humans with FXS (Loesch et al., 1988; Heulens et al., 2013) and indeed may be a factor in auditory hypersensitivity that has been underexplored. Consistent with our previous work, we see no alterations in pinna or facial characteristics in the C57BL/6J mouse model of FXS (McCullagh et al., 2020a) using calipers as a measurement tool. Others have explored morphological skull differences in FXS mice using different tools such as CT/MRI (Ellegood et al., 2010) and micro-CT (Heulens et al., 2013) with mixed results. Heulens et al., 2013 showed alterations in skull and jaw characteristics that had not been characterized previously with a similar technique (Ellegood et al., 2010) though differences may be due to how features were measured. We also see no difference in weight of Fmr1 animals compared to wildtype which is in contrast to our previous work where we noted that Fmrl animals weighed less than wildtype (McCullagh et al., 2020a) and others that showed an increase in male Fmr1 mouse weight compared to wildtype (Leboucher et al., 2019). Differences in weight may be due to inclusion of female animals (McCullagh et al., 2020a) and older animals (Leboucher et al., 2019). Overall changes in pinna morphology may still be an important factor in sound localization ability in Fmr1 animals and should be explored with more detailed techniques to determine if increased pinna measures in both humans and animal models may underly some aspect of auditory hypersensitivity symptomology.

384 Our previous results showed increased ABR measured hearing thresholds at high frequencies (16 $\mathrm{kHz}$ ) in the C57BL/6J Fmr1 strain with data combined for the sexes (McCullagh et al., 2020a). In the current study, we do not see increased thresholds at $16 \mathrm{kHz}$ but do see similar increased thresholds at $64 \mathrm{kHz}$ in male Fmr1 mice specifically. These data are consistent with the increased thresholds across frequencies seen in adult male FVB Fmrl mice (Rotschafer et al., 2015), though note that there was no change in threshold across frequencies in males of the same FVB strain at younger ages (Kim et al., 2013; El-Hassar et al., 2019). These data suggest that there may be age-related changes in high frequency hearing in adult male mice with FXS mutations across strains. This is particularly interesting since FVB mice are largely protected from early onset age-related hearing loss that the C57BL/6J background strain is particularly known for (Ison et al., 2007; Ho et al., 2014) suggesting that high frequency hearing deficits in males is a potentially conserved trait in FXS independent of background strain. Additional studies should examine hearing range across development and sexes in both strains to further show whether loss of high frequency hearing is a conserved feature in FXS.

Previous studies in the FVB Fmr1 mouse line show a robust wave I amplitude decrease in males across ages (Rotschafer et al., 2015; El-Hassar et al., 2019), though see (Kim et al., 2013). We do not see any change in wave I amplitude in the C57BL/6J Fmr1 line in adult animals of either sex. These conflicting results may be in part due to the earlier onset age-related hearing loss, which can be seen as decreases in early waves of the ABR, that occurs in the B6 background (Hunter and Willott, 1987). Changes in wave I amplitude specific to FXS may be masked by overall decreases in wave I 
amplitude across genotypes in this background. Interestingly, data in male FVB Fmr1 mice show no differences (adults, Kim et al., 2013; Rotschafer et al., 2015), or increased amplitudes in wave IV of the ABR (young, El-Hassar et al., 2019) whereas our data show decreased wave IV amplitude in Fmr1 females on the B6 background. These differences again may be due to differences in sexes and ages of animals tested. Lastly, our finding of no difference in latency of monaural waves is consistent with the majority of the work in FVB mice (Rotschafer et al., 2015; El-Hassar et al., 2019), though note that Kim et al., 2013 showed shorter latency for wave I. Our data further adds to the knowledge of ABR phenotypes that might be consistent across genotypes.

411 While ours is the first study to characterize the BIC in a FXS mutant mouse strain, our data are consistent with the BIC as it varies with ITD in mice (Benichoux et al., 2018). Namely, mice have a small range of ITD cues available due to their small head size and therefore the BIC amplitude decreases with increasing ITD between the ears, but this overall amplitude change is smaller than animals with more dominant ITD hearing ability (such as chinchilla or cats)(Benichoux et al., 2018). Additionally, consistent with previous work, the BIC latency gets longer with increasing ITD (Ferber et al., 2016; Laumen et al., 2016; Benichoux et al., 2018). Interestingly our work in FXS mice is consistent with increased latency of the BIC seen in a study in autistic people (ElMoazen et al., 2019), though they also see a decrease in amplitude of the BIC. Our findings that the BIC latency is only significant in males at 0 ITD potentially suggests that there is overall slowing of binaural processing in the brainstem, but that it is not dependent on ITD, which would be consistent with mice that do not rely as predominantly on ITD cues compared to other species.

The subject of sex differences in animal models is important for fully understanding the complexities of disorders such as autism spectrum disorder or FXS which seem to impact females differently than males (Werling and Geschwind, 2013; Nolan et al., 2017). In FXS, due to it being an X-linked disorder, there is a higher prevalence in males than females, which can undergo X-inactivation on the effected X chromosome (genetic mosaicism)(Kirchgessner et al., 1995). However, mice offer a unique opportunity to measure both heterozygote and homozygous females giving insight into potential sex differences related to loss of Fmrl on one or both X chromosomes. Our data suggest that there are indeed differences in auditory phenotypes between heterozygous and homozygous females (wave IV amplitude) in addition to differences between males and females. These and future data comparing female Fmrl subtypes may give insight into the role of X-inactivation in auditory brainstem processing phenotypes.

434 In conclusion, this study offers important insight into auditory phenotypes that may be shared or full or heterozygote mutation-specific differences in auditory brainstem function for both monaural and binaural hearing in B6 mice. Further studies measuring auditory phenotypes for B6 mice in earlier ages across the sexes would be useful to further characterize potential similarities with the FVB Fmr1 strain. In addition, characterizing the BIC in the FVB strain would be useful to elucidate if latency phenotypes are consistent across backgrounds.

\section{Conflict of Interest}

442 The authors declare that the research was conducted in the absence of any commercial or financial 443 relationships that could be construed as a potential conflict of interest. 
445

446

447

448

449

450

451

452

453

454

455

456

457

458

459

460

461

462

463

464

465

466

467

468

469

470

471

472

473

474

475

476

477

478

479

480

All authors helped write and revise the manuscript. EAM developed the ideas and methods. EAM and $\mathrm{AC}$ collected the data for the manuscript. EAM performed the statistical analyses and created the figures for the manuscript.

\section{Funding}

Supported by NIH 1R15HD105231-01. Preliminary work was also funded by a FRAXA research grant and NIH 3T32DC012280-05S1.

\section{Acknowledgements}

We would like to thank members of the McCullagh lab on team mouse that assisted with ABRs including Ishani Ray and Sabiha Alam. Further we would like to acknowledge Shani Poleg and Daniel Tollin for helping us set up these experiments in Colorado and continue them in Oklahoma.

\section{References}

Abbeduto, L., and Hagerman, R. J. (1997). Language and communication in fragile X syndrome. Mental Retardation and Developmental Disabilities Research Reviews 3, 313-322. doi:10.1002/(SICI)1098-2779(1997)3:4<313::AID-MRDD6>3.0.CO;2-O.

Alvarado, J. C., Fuentes-Santamaría, V., Jareño-Flores, T., Blanco, J. L., and Juiz, J. M. (2012). Normal variations in the morphology of auditory brainstem response (ABR) waveforms: a study in Wistar rats. Neurosci Res 73, 302-311. doi:10.1016/j.neures.2012.05.001.

Anbuhl, K. L., Benichoux, V., Greene, N. T., Brown, A. D., and Tollin, D. J. (2017). Development of the head, pinnae, and acoustical cues to sound location in a precocial species, the guinea pig (Cavia porcellus). Hear Res 356, 35-50. doi:10.1016/j.heares.2017.10.015.

Arnett, M. T., Herman, D. H., and McGee, A. W. (2014). Deficits in Tactile Learning in a Mouse Model of Fragile X Syndrome. PLoS ONE 9, e109116. doi:10.1371/journal.pone.0109116.

Bates, D., Mächler, M., Bolker, B., and Walker, S. (2015). Fitting Linear Mixed-Effects Models Using lme4. Journal of Statistical Software 67, 1-48. doi:10.18637/jss.v067.i01.

Beebe, K., Wang, Y., and Kulesza, R. (2014). Distribution of fragile X mental retardation protein in the human auditory brainstem. Neuroscience 273, 79-91. doi:10.1016/j.neuroscience.2014.05.006.

Benichoux, V., Ferber, A., Hunt, S., Hughes, E., and Tollin, D. (2018). Across Species "Natural Ablation" Reveals the Brainstem Source of a Noninvasive Biomarker of Binaural Hearing. The Journal of Neuroscience 38, 8563-8573. doi:10.1523/JNEUROSCI.1211-18.2018.

Beutelmann, R., Laumen, G., Tollin, D., and Klump, G. M. (2015). Amplitude and phase equalization of stimuli for click evoked auditory brainstem responses. J Acoust Soc Am 137, EL71-EL77. doi:10.1121/1.4903921.

Brittan-Powell, E. F., and Dooling, R. J. (2004). Development of auditory sensitivity in budgerigars (Melopsittacus undulatus). The Journal of the Acoustical Society of America 115, 3092-3102. doi:10.1121/1.1739479. 
481

482

483

484

485

486

487

488

489

490

491

492

493

494

495

496

497

498

499

500

501

502

503

504

505

506

507

508

509

510

511

512

513

514

515

516

517

Bronkhorst, A. W. (2015). The cocktail-party problem revisited: early processing and selection of multi-talker speech. Atten Percept Psychophys 77, 1465-1487. doi:10.3758/s13414-0150882-9.

Brown, M. R., Kronengold, J., Gazula, V.-R., Chen, Y., Strumbos, J. G., Sigworth, F. J., et al. (2010). Fragile $X$ mental retardation protein controls gating of the sodium-activated potassium channel Slack. Nature Neuroscience 13, 819-821. doi:10.1038/nn.2563.

Butler, R. A. (1975). "The Influence of the External and Middle Ear on Auditory Discriminations," in Auditory System: Physiology (CNS) - Behavioral Studies Psychoacoustics Handbook of Sensory Physiology., eds. M. Abeles, Gö. Bredberg, R. A. Butler, J. H. Casseday, J. E. Desmedt, I. T. Diamond, et al. (Berlin, Heidelberg: Springer), 247-260. doi:10.1007/978-3642-65995-9_6.

Chen, L., and Toth, M. (2001). Fragile X mice develop sensory hyperreactivity to auditory stimuli. Neuroscience 103, 1043-1050.

Curry, R. J., Peng, K., and Lu, Y. (2018). Neurotransmitter- and Release-Mode-Specific Modulation of Inhibitory Transmission by Group I Metabotropic Glutamate Receptors in Central Auditory Neurons of the Mouse. The Journal of Neuroscience 38, 8187-8199. doi:10.1523/JNEUROSCI.0603-18.2018.

Dahlhaus, R. (2018). Of Men and Mice: Modeling the Fragile X Syndrome. Front Mol Neurosci 11. doi:10.3389/fnmol.2018.00041.

El-Hassar, L., Song, L., Tan, W. J. T., Large, C. H., Alvaro, G., Santos-Sacchi, J., et al. (2019). Modulators of Kv3 Potassium Channels Rescue the Auditory Function of Fragile X Mice. The Journal of Neuroscience 39, 4797-4813. doi:10.1523/JNEUROSCI.0839-18.2019.

Ellegood, J., Pacey, L. K., Hampson, D. R., Lerch, J. P., and Henkelman, R. M. (2010). Anatomical phenotyping in a mouse model of fragile $\mathrm{X}$ syndrome with magnetic resonance imaging. Neuroimage 53, 1023-1029. doi:10.1016/j.neuroimage.2010.03.038.

ElMoazen, D., Sobhy, O., Abdou, R., and Ismail, H. (2019). Binaural Interaction Component of the Auditory Brainstem Response in Children with Autism Spectrum Disorder. International Journal of Pediatric Otorhinolaryngology, 109850. doi:10.1016/j.ijporl.2019.109850.

Ethridge, L. E., White, S. P., Mosconi, M. W., Wang, J., Pedapati, E. V., Erickson, C. A., et al. (2017). Neural synchronization deficits linked to cortical hyper-excitability and auditory hypersensitivity in fragile X syndrome. Molecular Autism 8. doi:10.1186/s13229-017-0140-1.

Ferber, A. T., Benichoux, V., and Tollin, D. J. (2016). Test-Retest Reliability of the Binaural Interaction Component of the Auditory Brainstem Response. Ear Hear 37, e291-301. doi:10.1097/AUD.0000000000000315.

Garcia-Pino, E., Gessele, N., and Koch, U. (2017). Enhanced Excitatory Connectivity and Disturbed Sound Processing in the Auditory Brainstem of Fragile X Mice. J. Neurosci. 37, 7403-7419. doi:10.1523/JNEUROSCI.2310-16.2017. 
518 Grothe, B., Pecka, M., and McAlpine, D. (2010). Mechanisms of sound localization in mammals.

519 Physiol. Rev. 90, 983-1012. doi:10.1152/physrev.00026.2009.

520 Hagerman, R. J., and Hagerman, P. J. (2002). Fragile X Syndrome: Diagnosis, Treatment and Research. Baltimore: Johns Hopkins University Press.

Heulens, I., Suttie, M., Postnov, A., De Clerck, N., Perrotta, C. S., Mattina, T., et al. (2013). Craniofacial characteristics of fragile X syndrome in mouse and man. Eur J Hum Genet 21, 816-823. doi:10.1038/ejhg.2012.265.

Hunter, K. P., and Willott, J. F. (1987). Aging and the auditory brainstem response in mice with severe or minimal presbycusis. Hear Res 30, 207-218. doi:10.1016/0378-5955(87)90137-7.

Ho, M. K., Li, X., Wang, J., Ohmen, J. D., and Friedman, R. A. (2014). FVB/NJ mice demonstrate a youthful sensitivity to noise-induced hearing loss and provide a useful genetic model for the study of neural hearing loss. Audiol Neurotol Extra 4, 1-11. doi:10.1159/000357770.

Ison, J. R., Allen, P. D., and O’Neill, W. E. (2007). Age-Related Hearing Loss in C57BL/6J Mice has both Frequency-Specific and Non-Frequency-Specific Components that Produce a Hyperacusis-Like Exaggeration of the Acoustic Startle Reflex. J Assoc Res Otolaryngol 8, 539-550. doi:10.1007/s10162-007-0098-3.

Kim, H., Gibboni, R., Kirkhart, C., and Bao, S. (2013). Impaired Critical Period Plasticity in Primary Auditory Cortex of Fragile X Model Mice. J Neurosci 33, 15686-15692. doi:10.1523/JNEUROSCI.3246-12.2013.

Kirchgessner, C. U., Warren, S. T., and Willard, H. F. (1995). X inactivation of the FMR1 fragile X mental retardation gene. J Med Genet 32, 925-929.

Laumen, G., Ferber, A. T., Klump, G. M., and Tollin, D. J. (2016). The Physiological Basis and Clinical Use of the Binaural Interaction Component of the Auditory Brainstem Response. Ear Hear 37, e276-90. doi:10.1097/AUD.0000000000000301.

Leboucher, A., Bermudez-Martin, P., Mouska, X., Amri, E.-Z., Pisani, D. F., and Davidovic, L. (2019). Fmr1-Deficiency Impacts Body Composition, Skeleton, and Bone Microstructure in a Mouse Model of Fragile X Syndrome. Front Endocrinol (Lausanne) 10, 678. doi:10.3389/fendo.2019.00678.

Levine, R. A. (1981). Binaural interaction in brainstem potentials of human subjects. Annals of Neurology 9, 384-393. doi:10.1002/ana.410090412.

Loesch, D. Z., Lafranchi, M., and Scott, D. (1988). Anthropometry in Martin-Bell syndrome. Am J Med Genet 30, 149-164. doi:10.1002/ajmg.1320300113.

Lu, Y. (2019). Subtle differences in synaptic transmission in medial nucleus of trapezoid body neurons between wild-type and Fmr1 knockout mice. Brain Research 1717, 95-103. doi:10.1016/j.brainres.2019.04.006. 
McCullagh, E. A., Poleg, S., Greene, N. T., Huntsman, M. M., Tollin, D. J., and Klug, A. (2020a). Characterization of Auditory and Binaural Spatial Hearing in a Fragile X Syndrome Mouse Model. eNeuro 7. doi:10.1523/ENEURO.0300-19.2019.

McCullagh, E. A., Rotschafer, S. E., Auerbach, B. D., Klug, A., Kaczmarek, L. K., Cramer, K. S., et al. (2020b). Mechanisms underlying auditory processing deficits in Fragile X syndrome. The FASEB Journal 00, 1-18. doi:https://doi. org/10.1096/fj.201902435R.

McCullagh, E. A., Salcedo, E., Huntsman, M. M., and Klug, A. (2017). Tonotopic alterations in inhibitory input to the medial nucleus of the trapezoid body in a mouse model of Fragile X syndrome. Journal of Comparative Neurology 262, 375. doi:10.1002/cne.24290.

Musicant, A. D., and Butler, R. A. (1984). The influence of pinnae-based spectral cues on sound localization. The Journal of the Acoustical Society of America 75, 1195-1200. doi:10.1121/1.390770.

New, E. M., Li, B.-Z., Lei, T., and McCullagh, E. A. (2021). Hearing Ability of Prairie Voles (Microtus ochrogaster). doi:10.1101/2021.10.07.463519.

Nolan, S. O., Reynolds, C. D., Smith, G. D., Holley, A. J., Escobar, B., Chandler, M. A., et al. (2017). Deletion of Fmr1 results in sex-specific changes in behavior. Brain and Behavior 7 , e00800. doi:10.1002/brb3.800.

R Core Team (2013). R: A Language and Environment for Statistical Computing. Vienna, Austria: R Foundation for Statistical Computing Available at: http://www.R-project.org/.

Radziwon, K. E., June, K. M., Stolzberg, D. J., Xu-Friedman, M. A., Salvi, R. J., and Dent, M. L. (2009). Behaviorally measured audiograms and gap detection thresholds in CBA/CaJ mice. $J$ Comp Physiol A Neuroethol Sens Neural Behav Physiol 195, 961-969. doi:10.1007/s00359009-0472-1.

Rotschafer, S. E., and Cramer, K. S. (2017). Developmental Emergence of Phenotypes in the Auditory Brainstem Nuclei of Fmr1Knockout Mice. eNeuro 4, 1-21. doi:10.1523/ENEURO.0264-17.2017.

Rotschafer, S. E., Marshak, S., and Cramer, K. S. (2015). Deletion of Fmr1 Alters Function and Synaptic Inputs in the Auditory Brainstem. PLoS ONE 10, 1-15. doi:10.1371/journal.pone.0117266.

Santos, M., Marques, C., Nóbrega Pinto, A., Fernandes, R., Coutinho, M. B., and Almeida E Sousa, C. (2017). Autism spectrum disorders and the amplitude of auditory brainstem response wave I. Autism Res 10, 1300-1305. doi:10.1002/aur.1771.

Stefanelli, A. C. G. F., Zanchetta, S., Furtado, E. F., Stefanelli, A. C. G. F., Zanchetta, S., and Furtado, E. F. (2020). Auditory hyper-responsiveness in autism spectrum disorder, terminologies and physiological mechanisms involved: systematic review. CoDAS 32 . doi:10.1590/2317-1782/20192018287. 
The Dutch-Belgian Fragile X Consorthium, Bakker, C. E., Verheij, C., Willemsen, R., van der Helm, R., Oerlemans, F., et al. (1994). Fmr1 knockout mice: A model to study fragile X mental retardation. Cell 78, 23-33. doi:10.1016/0092-8674(94)90569-X.

Tian, Y., Yang, C., Shang, S., Cai, Y., Deng, X., Zhang, J., et al. (2017). Loss of FMRP Impaired Hippocampal Long-Term Plasticity and Spatial Learning in Rats. Front Mol Neurosci 10, 269. doi:10.3389/fnmol.2017.00269.

Till, S. M., Asiminas, A., Jackson, A. D., Katsanevaki, D., Barnes, S. A., Osterweil, E. K., et al. (2015). Conserved hippocampal cellular pathophysiology but distinct behavioural deficits in a new rat model of FXS. Hum Mol Genet 24, 5977-5984. doi:10.1093/hmg/ddv299.

Visser, E., Zwiers, M. P., Kan, C. C., Hoekstra, L., van Opstal, A. J., and Buitelaar, J. K. (2013). Atypical vertical sound localization and sound-onset sensitivity in people with autism spectrum disorders. J Psychiatry Neurosci 38, 398-406. doi:10.1503/jpn.120177.

Wang, T., de Kok, L., Willemsen, R., Elgersma, Y., and Borst, J. G. G. (2015). In vivo synaptic transmission and morphology in mouse models of Tuberous sclerosis, Fragile $\mathrm{X}$ syndrome, Neurofibromatosis type 1, and Costello syndrome. Front Cell Neurosci 9, 234. doi:10.3389/fncel.2015.00234.

Wang, Y., Sakano, H., Beebe, K., Brown, M. R., de Laat, R., Bothwell, M., et al. (2014). Intense and specialized dendritic localization of the fragile $\mathrm{X}$ mental retardation protein in binaural brainstem neurons: A comparative study in the alligator, chicken, gerbil, and human: FMRP localization in NL/MSO dendrites. Journal of Comparative Neurology 522, 2107-2128. doi:10.1002/cne.23520.

Werling, D. M., and Geschwind, D. H. (2013). Sex differences in autism spectrum disorders. Curr Opin Neurol 26, 146-153. doi:10.1097/WCO.0b013e32835ee548.

Wickham, H. (2016). ggplot2: Elegant Graphics for Data Analysis. Springer-Verlag New York Available at: http://ggplot2.org. 